\title{
Multi-Source and Multi-Scale Platform for Quantitative Assessment of Shallow or/and Coastal Qater ${ }^{+}$
}

\author{
Anna Brook ${ }^{1, *}$, Ran Reznikov ${ }^{1}$, Martin Kanning ${ }^{2}$ and Thomas Jarmer ${ }^{2}$ \\ 1 Department of Geography and Environmental Studies, University of Haifa, Haifa 3498838, Israel; \\ ranner21@gmail.com \\ 2 Institute for Geoinformatics and Remote Sensing, University of Osnabrück, 49074 Osnabrück, Germany; \\ makanning@uni-osnabrueck.de (M.K.); tjarmer@igf.uni-osnabrueck.de (T.J.) \\ * Correspondence: abrook@geo.haifa.ac.il \\ † Presented at TERRAenVISION 2019, Barcelona, Spain, 2-7 September 2019.
}

Published: 15 November 2019

\begin{abstract}
Coastal waters are one of the most vulnerable resources that require comprehensive investigation in space and time. One of the key factors for effective coastal monitoring is the use of remote sensing technologies. Since the Coastal Zone Color Scanner (CZCS) in 1978, a long list of space-borne missions had been successfully launched. However, those missions are limited to coastal waters applications. Despite a large number of missions, the existing systems are still facing similar challenges as four decades ago. Spatial and spectral data reconstruction and recovery a high resolution (HR) imagery data from a low resolution (LR) imaging is a challenging task in many applications. The most promising technique in the field of digital image processing is known as Super Resolution (SR). Many techniques focus on reconstructing information at the sub-pixel level and dividing the original LR space into pixels corresponding to the HR space. Other methods assume that a series of LR images (in time) of a scene scanned from different perspectives (affine) will provide SR. Alternative methods use different data sources and proper image algorithms. In most cases, SR methods will perform a learning process in which the system will try to identify the inherent redundancy in the natural data in order to retrieve HR information from LR based on a spatial correlation between the original images. The learning process can be significantly efficient by using the Convolutional Neural Network (CNN). CNN submit to training through a large dataset that preserves the scene's characteristics. The flexibility afforded by CNN is learning nonlinear relationships when reconstructing a spatial characteristic from an LR image to HR image. The main aim of this study is to identify spectral features related to the coastal water and inland water variations at different spatial and temporal scale and integrate them with a multi-scale information system. The main objectives of the study are developing of spatial-temporal-spectral fusion approach for multi-source data collected from the same geographical site; creating a new method for single image reconstruction from non-complementary information scene. The proposed method measures HR given LR by a downscaling process by turning HR into an LR. The deterministic process calculated using a Gaussian filter and by a photographic-focused distribution function. The correlation coefficient (at the LR-pixel level) used as an inverse ratio to upscaling. The proposed architecture is based on a three-convolutional network. In the first stage, the convolution is directly applied to the LR data, and then another sub-pixel convolution layer is subtracted to generate SR data from LR data through an upscaling process. This study performed in two sites, (1) a training site in Israel, (2) a test site in Germany. The training site is shallow seawaters around Oren River, Israel and the test site is Alfsee inland water in Germany. The results in both sites are SR imagery with full Sentinel 2 spectral resolution and spatial resolution of $0.3 \mathrm{~m}$.
\end{abstract}


Keywords: coastal waters; Pan-sharpening; UAV; RapidEye 